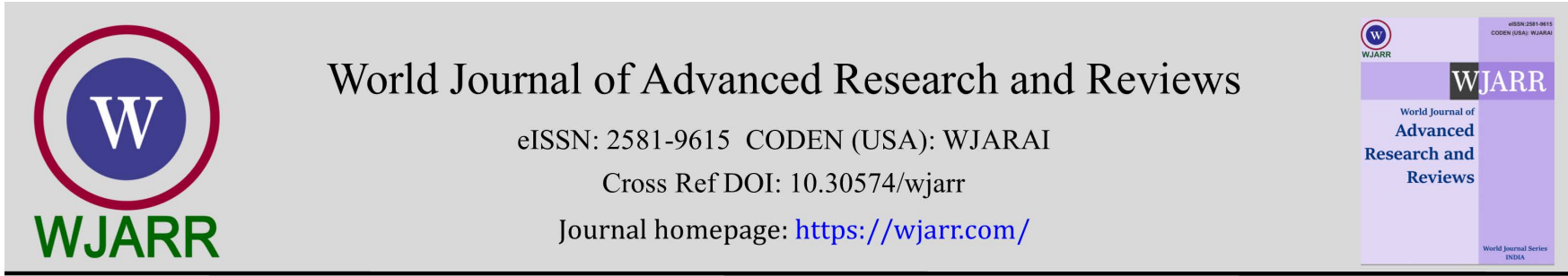

(CASE REPORT)

Check for updates

\title{
Primary Mediastinal Large B-cell Lymphoma Treatment and Prevention of Anthracycline Cardiotoxicity: A Case Report
}

\author{
A Ter-Grigoryan, P Ghazaryan, N Melkikyan and L Evoyan * \\ Center of Haematology named after prof. R.H. Yeolyan, Armenia.
}

World Journal of Advanced Research and Reviews, 2021, 10(01), 132-135

Publication history: Received on 02 March 2021; revised on 04 April 2021; accepted on 07 April 2021

Article DOI: https://doi.org/10.30574/wjarr.2021.10.1.0142

\begin{abstract}
Primary mediastinal B-cell lymphoma (PMBCL) is a relatively rare lymphoma subtype affecting mainly young adults. Its molecular signature and clinical features resemble classical Hodgkin lymphoma. The optimal chemotherapy for this lymphoma subtype has not been established. The addition of rituximab to anthracycline based chemotherapy improved response rates and survival. Many centers use R-CHOP as standard treatment, but the role of the intensified regimens and consolidation radiotherapy has to be clarified. Recent data coming from retrospective analyses and an ongoing prospective study addressing the problem of consolidation radiotherapy will help to better identify risk groups and apply risk-adapted and effective treatment strategies. The latest research has helped to understand molecular mechanisms of PMBCL pathogenesis and indicated targets of directed therapy for the future.
\end{abstract}

Keywords: Primary Mediastinal B-Cell Lymphoma; Anthracycline Cardiotoxicity; Invasive Thymoma; Doxorubicin Cumulative Dose; Mediastinal Mass.

\section{Introduction}

Primary mediastinal B-cell lymphoma (PMBCL) belongs to the group of aggressive diffuse large B-cell lymphomas. The current WHO 2008 classification distinguished this lymphoma as a separate entity due to its specific clinical and pathological features [1]. Gene expression profile studies showed that it shares common features with classical Hodgkin lymphoma (cHL) $[2,3]$. Treatment outcomes in the pre-rituximab era were not satisfactory with high relapse rates. Adding rituximab to anthracycline based regimens improved patient prognosis, and R-CHOP has been widely adopted as the standard treatment. However, there are still unresolved questions in the therapy of PMBCL. There are questions such as is R-CHOP an optimal regimen for all patients? Historical data indicated the superiority of more intense chemotherapy regimens, but they have not been compared to R-CHOP directly, and there is no consensus which group of patients would benefit from intensified regimens. Also, there is no convincing data supporting the use of radiotherapy. Retrospective studies generally did not show survival benefit, and late toxicities like cardiotoxicity and secondary cancers cannot be neglected. However, the questions are if radiotherapy can be safely omitted and in which patient group it can be omitted, so far have not been answered. Relatively low patient numbers are the main obstacle in conducting randomized prospective trials, so therapeutic decisions have been based mainly on retrospective studies.

\section{Case presentation}

We present a case of a 36-year-old female patient with a PMBCT that was previously misdiagnosed and treated as invasive thymoma.

\footnotetext{
${ }^{*}$ Corresponding author: L Evoyan

Hematology center after prof. R.H. Yeolyan, Yerevan, Armenia.

Copyright $(2021$ Author(s) retain the copyright of this article. This article is published under the terms of the Creative Commons Attribution Liscense 4.0.
} 
The patient presented to an Armenian National Center of Oncology after V. A. Fanarjyan in November 2019 with a 3month history of weakness, prolonged fever (a recurrent fever type, with a temperature of $>380 \mathrm{C}$ for 1-2 days before returning to normal and recurring repeatedly over 3 months), night sweats, accompanied by chest pain. The physical examination did not reveal any abnormalities and the patient had no history of a similar disease or any hereditary disorders in her family.

Upon presentation, the blood cell count was normal, the liver and renal function tests were almost normal, while the biochemical evaluations revealed signs of inflammation. Computed tomography (CT) with contrast identified anterior mediastinal mass of $9.5 \times 10.3 \times 7.8 \mathrm{~cm}$ with the invasion of the pericardium and compressive atelectasis of the upper lobe of the left lung (anterior S3 and S4 segments), as well mediastinal lymphadenopathy up to 2-3cm without pulmonary trunk involvement. The liver and spleen were not enlarged. A needle biopsy was performed by a parasternal approach and the results of the pathological examination revealed thymic carcinoma. The patient underwent 7 courses of polychemotherapy (doxorubicin 80mg IV + cyclophosphamide 850mg IV + cisplatinum 80mg IV). After all 7 courses, contrast-enhanced CT identified that mediastinal mass persisted (anterior mediastinal mass $5.6 \times 6.0 \times 5.8 \mathrm{~cm}$, mediastinal lymphadenopathy up to $1.5 \mathrm{~cm}$ ). Thus, it was decided to perform radical surgery, i.e., thymectomy, left upper lobectomy with pulmonary artery reconstruction. The following pathological examination and immunohistochemistry staining results led to a diagnosis of primary mediastinal (thymic) large B-cell lymphoma (PMLBCL): sIG-/+, PanB+ (especially CD20, CD79a), CD45+/-, CD15-, CD30-/+ (weak), IRF4/MUM1 +/-, BCL2+/-, BCL6 +/-, CD23+, MAL+, IGH and IGL gene rearrangements, Ki67=90\%.
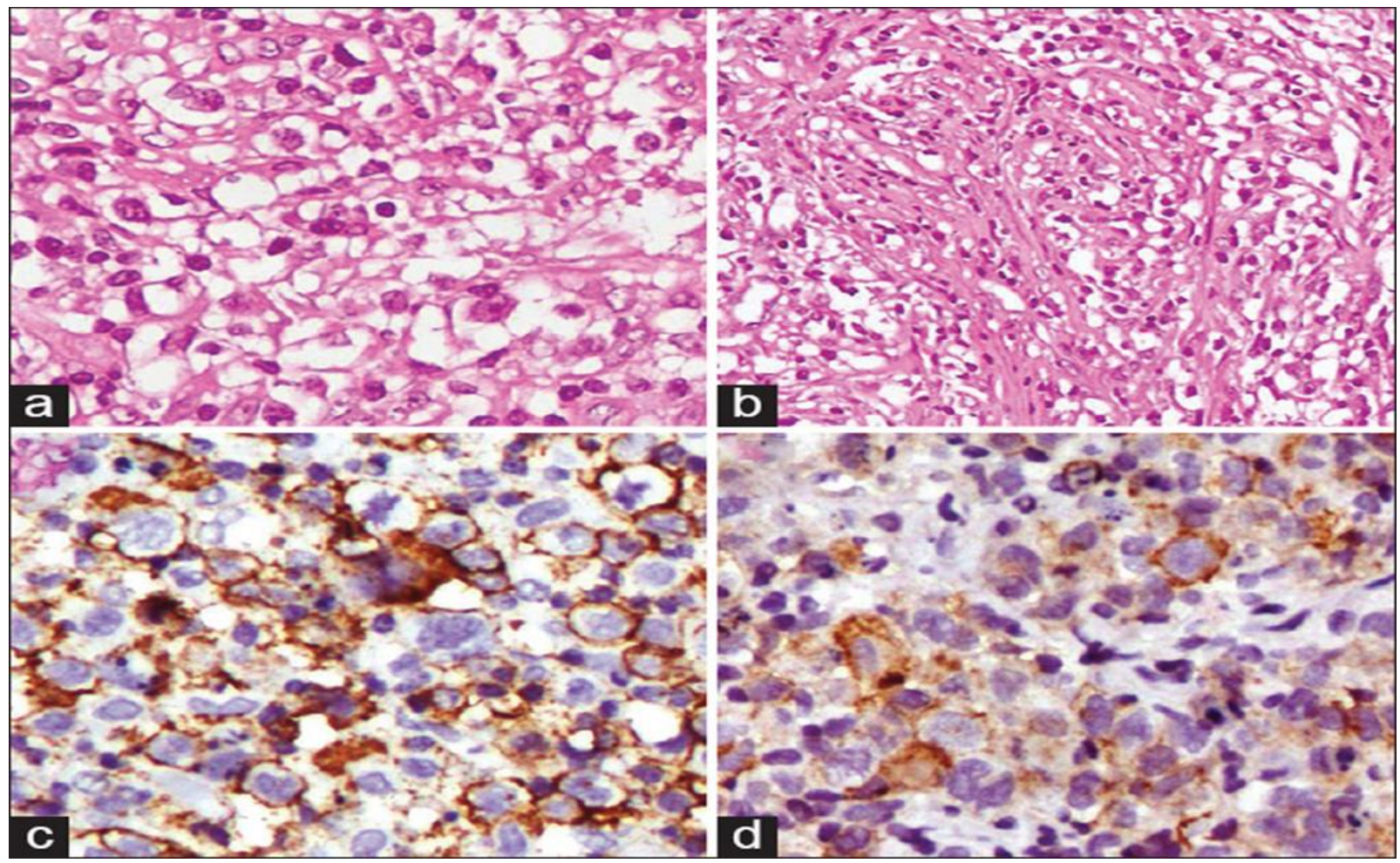

Figure 1 Primary mediastinal B cell lymphoma. (a) tumor cells are round to ovoid with abundant clear cytoplasm (b) compartmentalizing fibrosis (c) diffuse and strong CD20 positivity (d) and heterogenous CD30 positivity

The patient presented to Hematology Center after prof. R.H. Yeolyan in June 2020. Upon presentation patient's symptoms were weakness, fever, night sweats, and chest pain. The physical examination was mostly normal, with a normal appearance and range of motion. The blood cell count revealed white blood cells $7.4 \times 109 / \mathrm{l}$; band neutrophils $2.0 \%$, segmented neutrophils $60.0 \%$, lymphocytes 24.0 , eosinophils $2.0 \%$, monocytes $12.0 \%$, ESR 38mm/h, hemoglobin level and RET\% were normal (132g/l), platelets $316 \times 109 / \mathrm{l}$. The liver and renal function tests and the biochemical tests, and the coagulation screen were almost normal. The EF of the heart was in a normal range. The trephine biopsy was performed, and pathological examination didn't reveal any signs of bone marrow involvement. Therefore, the patient received 2 courses of R-CHOEP chemotherapy (rituximab 600mg IV, cyclophosphamide 1200mg IV, doxorubicin $80 \mathrm{mg}$ IV, vincristine $2 \mathrm{mg}$ IV on the first day, etoposide $150 \mathrm{mg}$ IV for two days, $200 \mathrm{mg}$ IV on the third day and prednisolone $70 \mathrm{mg}$ per os for 5 days). After one month, 18F-FDG positron emission tomography - computed tomography (PET/CT) was performed, which showed postoperative FDG-negative mediastinal changes (scary tissue), 
subcentimetric negative pulmonary nodules. There were no infiltrative focal changes in the lungs. The rest of the body was clear. The patient had also received consolidative 3D radiotherapy for the mediastinum. After the treatment the overall health condition of the patient is stable despite having rare chest pain. On January 25, 2021, whole-body CT was performed, and no sign of the disease was observed.

\section{Discussion}

In clinical practice, most clinicians limit the cumulative dose of doxorubicin to $400-450 \mathrm{mg} / \mathrm{m} 2$. As the patient has already received 7 courses of doxorubicin, cyclophosphamide, and cisplatine, it is crucial to consider the usual cumulative dose of doxorubicin in the further treatment of the patient with R-CHOP protocol. In this case after overall 9 courses of polychemotherapy the anthracycline cumulative dose is equals to $360 \mathrm{mg} / \mathrm{m} 2$. We also performed cardiac ultrasound which identified normal left ventricular ejection (55\%). We also performed a cardiac ultrasound which identified normal left ventricular ejection (55\%).

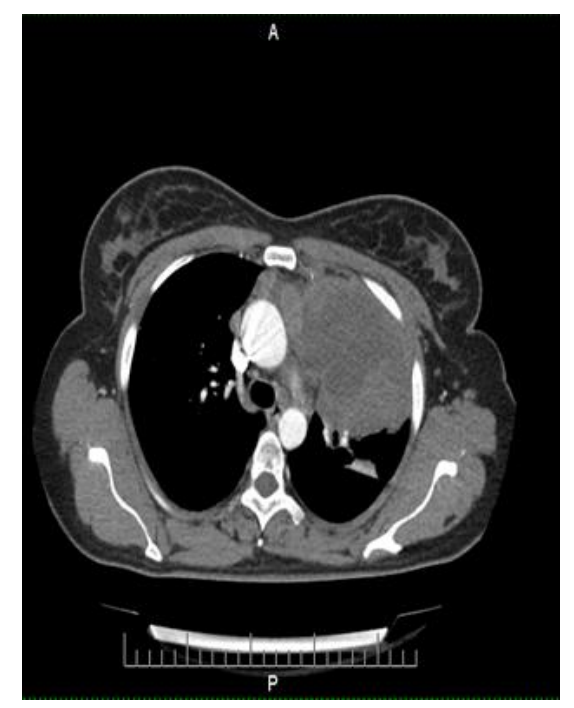

Pretreatment

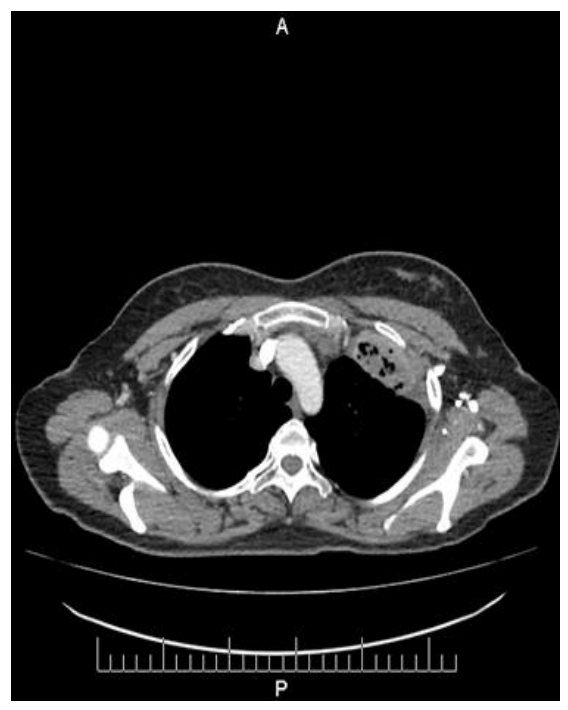

CT 9 cycles

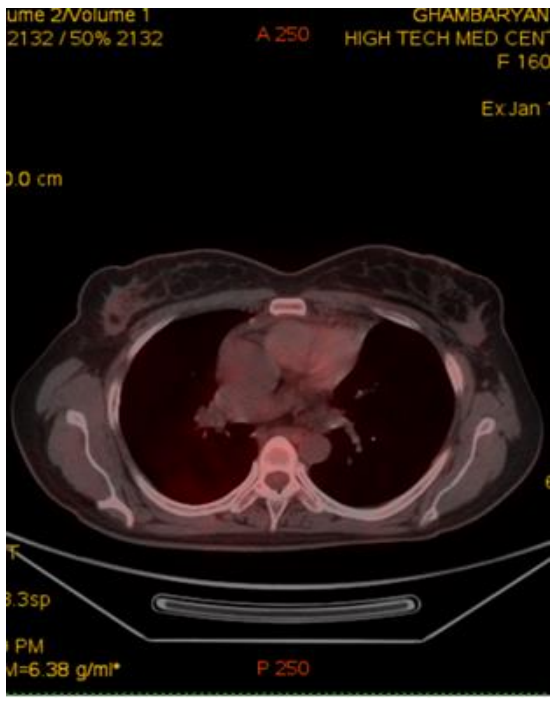

PET-CT 9 cycles

Figure 2 Imaging demonstrating response to treatment. CT and PET-CT after 9 cycles.

After two courses of R-CHOEP chemotherapy, we decided not to continue the chemotherapy and do only a consolidative $3 \mathrm{D}$ radiotherapy.

\section{Conclusion}

Thus, the present case study indicated the role of prevention of cardiac toxicity. Besides, to improve the success rate of diagnosis, it is important that physicians attempt to retrieve as much of the diseased tissue as possible from the multiple lesions. We also recommend conducting a second biopsy if the results of the first biopsy are suspicious.

\section{Compliance with ethical standards}

\section{Acknowledgments}

We thank the management of Hematology Center, as well as Anahit Ter-Grigoryan the head of Adult clinical hematology department for great support. We are also thankful to the patient and her family for their valuable support in the preparation of this manuscript.

\section{Disclosure of conflict of interest}

The authors have no conflict of interest to declare. 


\section{Statement of informed consent}

Written informed consent was obtained from patient in this study.

\section{References}

[1] Swerdlow SH, Campo E, Harris NL, Jaffe ES, Pileri S, Stein H, Thiele J, Vardiman J (eds). WHO Classification of Tumors of Haematopoietic and Lymphoid Tissues. 4th edn. Lyon: IARC. 2008; 250-251.

[2] Savage K, Monti S, Kutok JL, et al. The molecular signature of mediastinal large B-cell lymphoma differs from that of other diffuse large B-cell lymphomas and shares features with classical Hodgkin lymphoma. Blood. 2003; 102(12): 3871-9. [PubMed].

[3] Rosenwald A, Wright G, Leroy K, et al. Molecular diagnosis of primary mediastinal B cell lymphoma identifies a clinically favourable subgroup of diffuse large B cell lymphoma related to Hodgkin lymphoma. J Exp Med. 2003; 198(6): 851-62. 